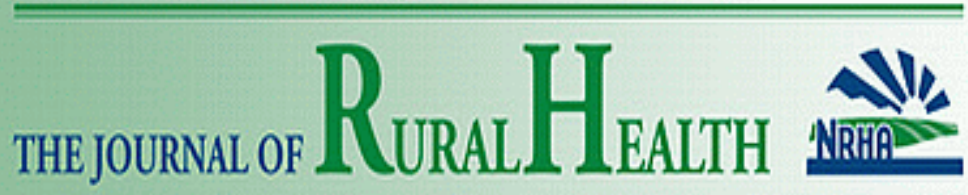

\title{
Rural-Urban Disparities in Total Physical Activity, Body Composition, and Related Health Indicators: An Atlantic PATH Study
}

\begin{tabular}{|c|c|}
\hline Journal: & The Journal of Rural Health \\
\hline Manuscript ID & $\mathrm{JRH}-2018-08-0208 . \mathrm{R} 2$ \\
\hline Wiley - Manuscript type: & Original Article \\
\hline Keywords: & obesity, cohort, rural-urban disparities, epidemiology, physical activity \\
\hline Abstract: & $\begin{array}{l}\text { Purpose: To describe and compare the sociodemographic and lifestyle } \\
\text { characteristics of urban and rural residents in Atlantic Canada. } \\
\text { Methods: Cross-sectional analyses of baseline data from the Atlantic } \\
\text { Partnership for Tomorrow's Health cohort was conducted. Specifically, } \\
17,054 \text { adults ( } 35-69 \text { years) who provided sociodemographic } \\
\text { characteristics, measures of obesity, and a record of chronic disease and } \\
\text { health behaviors were included in the analyses. Multiple linear regression } \\
\text { and logistic regression models were used to calculate the multivariable- } \\
\text { adjusted beta coefficients ( } \beta \text { ), odds ratios (OR) and related } 95 \% \\
\text { confidence intervals. } \\
\text { Findings: After adjusting for age, sex, and province, when compared to } \\
\text { urban participants, rural residents were significantly more likely to be } \\
\text { classified as very active (OR } 1.19[1.11-1.27]) \text {, obese (OR } 1.13 \text { [1.05- } \\
1.21]) \text {, to present with abdominal obesity (OR } 1.08 \text { [1.01-1.15]), higher } \\
\text { body fat percentage ( } \beta 0.40 \text { [ } 0.12-0.68]) \text {, and fat mass index ( } \beta 0.32 \\
\text { [0.19-0.46]). Rural residents were significantly less likely to be regular } \\
\text { or habitual drinkers (OR } 0.83 \text { [0.78-0.89]). Significant differences } \\
\text { remained after further adjustment for confounding sociodemographic, } \\
\text { lifestyle, and health characteristics. No significant differences in smoking } \\
\text { behavior, fruit and vegetable intake, multimorbidity or waist } \\
\text { circumference were found. } \\
\text { Conclusions: As expected, obesity prevalence was higher in rural Atlantic } \\
\text { Canadians. In contrast to much of the existing literature, we found that } \\
\text { rural participants were more likely to report higher levels of total } \\
\text { physical activity and lower alcohol consumption. Findings suggest that } \\
\text { novel obesity prevention strategies may be needed for rural populations. }\end{array}$ \\
\hline
\end{tabular}

\section{SCHOLARONE Manuscripts}


Forbes, Cynthia; Yu, Z. Michael; Cui, Yunsong; DeClercq, Vanessa; Grandy, Scott; Parker, Louise; Sweeney, Ellen; Dummer, Trevor; Keats, Melanie

\begin{abstract}
Purpose: To describe and compare the sociodemographic and lifestyle characteristics of urban and rural residents in Atlantic Canada.

Methods: Cross-sectional analyses of baseline data from the Atlantic Partnership for Tomorrow's Health cohort was conducted. Specifically, 17,054 adults (35-69 years) who provided sociodemographic characteristics, measures of obesity, and a record of chronic disease and health behaviors were included in the analyses. Multiple linear regression and logistic regression models were used to calculate the multivariable-adjusted beta coefficients $(\beta)$, odds ratios (OR) and related 95\% confidence intervals.

Findings: After adjusting for age, sex, and province, when compared to urban participants, rural residents were significantly more likely to be classified as very active (OR 1.19 [1.11-1.27]), obese (OR 1.13 [1.05-1.21]), to present with abdominal obesity (OR 1.08 [1.01-1.15]), higher body fat percentage ( $\beta 0.40$ [0.12-0.68]), and fat mass index ( $\beta 0.32$ [0.19-0.46]). Rural residents were significantly less likely to be regular or habitual drinkers (OR 0.83 [0.78-0.89]). Significant differences remained after further adjustment for confounding sociodemographic, lifestyle, and health characteristics. No significant differences in smoking behavior, fruit and vegetable intake, multimorbidity or waist circumference were found.
\end{abstract}

Conclusions: As expected, obesity prevalence was higher in rural Atlantic Canadians. In contrast to much of the existing literature, we found that rural participants were more likely to report higher levels of total physical activity and lower alcohol consumption. Findings suggest that novel obesity prevention strategies may be needed for rural populations.

This is the peer reviewed version of the following article: The journal of rural health, which has been published in final form at https://doi.org/10.1111/jrh.12363. This article may be used for non-commercial purposes in accordance with Wiley Terms and Conditions for Use of Self-Archived Versions. 


\section{Introduction}

Obesity is a growing health concern in many countries. ${ }^{1-7}$. Higher levels of body mass and more specifically, body fat mass, are associated with numerous chronic conditions such as cardiovascular disease, diabetes mellitus, and many types of cancer. ${ }^{7-12}$ Escalating rates of obesity and related health conditions have resulted in significant financial strain on the healthcare systems of many developed countries. ${ }^{13}, 14$ The obesity epidemic and associated chronic diseases are frequently attributed to lifestyle behaviors including, but not limited to low levels of physical activity (PA), sedentary behavior, sleep, dietary choices, tobacco use and alcohol consumption. ${ }^{15}$ While important determinants of obesity, the assessment of lifestyle behaviors in isolation of an understanding of the broader contextual influences or distal risk factors (eg, physical and socioeconomic characteristics associated with rural/urban residence) may result in an incomplete understanding of the growing obesity epidemic. ${ }^{15}$

Research in developed countries indicates a trend towards rural residents generally having poorer health outcomes than those living in urban areas. For example, rural residents have been shown to be more likely to be obese and have a higher prevalence of chronic disease than their urban counterparts. ${ }^{16-19}$ Studies in the United States, ${ }^{1,10,13,16,19}$ Canada, ${ }^{20,21}$ Australia, ${ }^{18,22}$ and the United Kingdom ${ }^{4}, 14$ have all demonstrated this relationship. These differences in obesity and chronic disease may be due to the lifestyle behaviors that influence body mass as rural residents are more likely to be physically inactive, sedentary, have poorer diets, smoking and drinking behaviors. ${ }^{16-19}$ Moreover, research in this area suggests that differences in culture, ${ }^{23,24}$ socioeconomic status, ${ }^{21,25,26}$ and/or environmental conditions (eg, green space, access to recreational facilities, pathways, sidewalks, and parks) $)^{23,25,27}$ may be important in understanding 
the differences in obesity-related risk factors and associated health outcomes in urban and rural populations.

Approximately $20 \%$ of all Canadians are classified as living in a rural region; however, in Atlantic Canada, an average of $46 \%$ of the population are considered rural $(44.5 \%$ Nova Scotia, 49\% New Brunswick, 42.4\% Newfoundland and Labrador, and 55\% Prince Edward Island). ${ }^{28}$ Notably, compared to the rest of Canada, Atlantic Canadians also generally exhibit poorer health profiles and higher rates of chronic disease. ${ }^{29}$ While lifestyle risk factors are often described as the proximal precursors of disease, research has suggested that the social and economic disparities often encountered in rural populations are at the root of chronic disease. ${ }^{30}$ Accordingly, the purpose of the current study was to describe and compare the sociodemographic and lifestyle characteristics of Atlantic Canadians residing in rural versus urban populations. Based on previous research among developed countries, ${ }^{1,16-19}$ we hypothesized that those living in rural areas would have poorer health behaviors and higher rates of obesity and multimorbidity than those in urban areas.

\section{Methods}

Study Design

This study was a cross-sectional analysis of data from the Atlantic Partnership for Tomorrow's Health (Atlantic PATH), the Atlantic chapter of the Canadian Partnership for Tomorrow Project (CPTP). CPTP is a pan-Canadian prospective, longitudinal cohort study. A detailed description of the study has been previously published. ${ }^{31}$ The Atlantic PATH study aims to examine and better understand the interplay between modifiable and non-modifiable risk factors that contribute to the development of cancer and other chronic diseases in Atlantic 
Canada. ${ }^{32}$ Additionally, the larger CPTP study aims to determine the differences in the regional cohorts and how this may impact disease development and management.

Detailed data collection procedures have been previously described. ${ }^{32}$ In brief, between 2009-2015, 31,173 participants aged 35-69 years across all four Atlantic provinces completed a standardized set of questionnaires designed to assess sociodemographic characteristics, health status, disease history, and lifestyle behaviors (ie, diet, smoking, alcohol use, and PA). Measures of body composition were collected at an assessment center or mobile clinic by a research nurse. All participants provided informed consent. Participants with all relevant variables completed were included in the final analyses (ie, complete-case analysis), for a total of 17,054 complete cases.

Measures

Participant Characteristics

Demographic variables were used to describe the sample. For analyses, categorical variables were categorized as follows: ethnicity (white/non-white), education (high school or lower/college level/university level or higher), marital status (married or living together/single, divorced, separated, or widowed), smoking behavior (non-smoker/former smoker/current smoker), and alcohol consumption (abstainer/occasional drinker/regular drinker/habitual drinker). Multimorbidity was defined as having $\geq$ two self-reported chronic conditions from a list of 21 conditions assessed at baseline. ${ }^{33}$

\section{Anthropometrics and Body Adiposity}

A Tanita bioelectrical impedance device (Tanita BC-418, Tanita Corporation of America Inc., Arlington Heights, Illinois) was used to measure body weight (kg) and percentage fat mass $(\%)$, height $(\mathrm{cm})$ was measured with a Seca stadiometer. Waist and hip circumference $(\mathrm{cm})$ were 
measured using a standard measuring tape. Abdominal obesity was defined as a waist circumference $\geq 102 \mathrm{~cm}$ for men or $\geq 88 \mathrm{~cm}$ for women. ${ }^{34}$ Height and weight measures were used to calculate body mass index (BMI; weight in kilograms divided by height in meters squared; $\mathrm{kg} / \mathrm{m}^{2}$ ). Obesity was defined as a $\mathrm{BMI} \geq 30 \mathrm{~kg} / \mathrm{m}^{2}$. Fat mass index (FMI) and fat free mass index (FFMI; $\mathrm{kg} / \mathrm{m}^{2}$ ) was calculated by dividing fat mass and fat free mass in kilograms by height in meters squared, respectively.

Physical Activity and Sedentary Behaviors

Participants were asked to report their levels of PA and sitting time using open-ended questions in the International Physical Activity Questionnaire (IPAQ) long- and short-forms which asks participants to indicate the frequency and duration of activities over the past seven days. ${ }^{35,36}$ The IPAQ-Long Form (IPAQ-LF) specifies four activity domains, including occupational (ie, activity done while at work), transportation (ie, how they commuted to work), domestic (ie, time spent gardening or doing housework), and leisure-time (ie, any other activity completed not part of the other domains). A measure of sedentary behaviors (ie, time spent sitting and motor vehicle use) is also included. Within each domain, participants are asked to indicate for each intensity how many days in the last week they performed the activity (ie, frequency) and for how many hours and/or minutes each session lasted (ie, duration). The IPAQShort Form (IPAQ-SF) asks general questions regarding amounts of sitting, walking, moderate, and vigorous intensity activity accumulated in all aspects of daily living. Participants were asked to separately report how many hours and minutes per day were spent sitting on an average weekday and weekend day. Participants were asked only to report activities that were in bouts of 10 minutes or more. Each question defined the specific intensity and domain and provided examples. The IPAQ is a commonly used as a measure of population levels of PA and both the 
long and short forms have been shown to provide reliable and valid data that are comparable to other self-report measures. ${ }^{35,36}$

Using the IPAQ guidelines for data processing and analyses, ${ }^{37}$ daily and weekly metabolic equivalents of a task (MET) values were calculated using data from both the long and short forms. Total activity minutes and MET-minutes were then converted into hours and METhours. This method was chosen to reduce the number of missing cases as only $67 \%$ of the study sample had completed the IPAQ-LF. Depending on the time of study entry (2009-2015) not all participants had access to the IPAQ-LF. Overall, $67 \%$ of participants completed the LF.

As self-reported moderate-to-vigorous PA data was highly skewed in the current study, sex-specific, data driven tertiles were calculated for both the LF and SF to determine high, moderate, and low levels of activity. This method has been previously utilized to allow comparisons between participants who completed either the long or short form of the IPAQ. ${ }^{38}$ Diet

Measurement of fruit and vegetable consumption (including 100\% fruit and vegetable juice) was adapted from the Canadian Community Health Survey (Cycles 1.1 and 3.1). ${ }^{39,40}$ Total daily servings of fruit, vegetables, and $100 \%$ fruit or vegetable juice was assessed by the following three questions: 1) In a typical day, how many servings of vegetables do you eat? One serving is about $1 / 2$ cup or $125 \mathrm{ml}$ of fresh, frozen, canned or cooked vegetables; 2) In a typical day, how many servings of fruit do you eat? One serving is about $1 / 2$ cup or $125 \mathrm{ml}$ of fresh, frozen, or canned fruit; 3) On a typical day, how many servings of $100 \%$ fruit or vegetable juice do you drink? One serving is about $1 / 2$ cup or $125 \mathrm{ml}$. For the present study, the number daily servings of fruits, vegetables, and $100 \%$ fruit juices was summed. The combined value was expressed as the overall number of daily servings of fruit and vegetables. Adequate fruit and 
vegetable intake was then defined as consuming at least 7 servings of combined daily servings of fruits, vegetables, and 100\% fruit juices.

\section{Alcohol Consumption}

Measure of alcohol use was adapted from the Canadian Health Measures Survey (cycle 1 - Household Survey). ${ }^{41}$ Specifically, participants were asked to indicate whether they had ever consumed alcohol (yes/no). If so, they were asked to complete an addition item detailing the average frequency of alcohol consumption over the last year (never, less than monthly, about once a month, 2-3 times per month, once a week, 2-3 times per week, 4-5 times per week, 6-7 times per week). Respondents were then classified as abstainer, occasional drinker ( $>0$ to $\leq 2-3$ times/month), regular drinker $(\geq 1$ time/week to $\leq 2-3$ times/week), and habitual drinker ( $\geq 4-5$ times/week).

\section{Smoking Behavior}

Items adapted from the Canadian Health Measures Survey (Cycle 1 - Household Survey $)^{41}$ and the Canadian Tobacco Survey ${ }^{42}$ were also used to assess cigarette smoking behavior. Participants were first asked if they had smoked at least 100 cigarettes in their lifetime. If yes, they were asked to respond to additional questions as applicable including at what age they smoked their first whole cigarette, what their smoking behavior was at present, at what age they began daily smoking, how many cigarettes per day they smoke now (or did when a daily smoker), and for how many years they were a daily smoker. Participants were then categorized as non-smoker, former smoker, and current smoker.

\section{Urban or Rural Residency}

The Postal Code Conversion File Plus (PCCF+, version 6C, Statistics Canada) was used to classify study participants as living in urban or rural areas according to their reported 
residential postal code. ${ }^{26}$ The most recent file available based on year of baseline survey (20092015) was used when classifying participants as rural or urban since the community status may have changed from the time they filled out the baseline questionnaire to time of analysis. Analyses

Descriptive statistics including counts, percentages, means and standard deviations were used to describe the sample. Preliminary groups differences between urban and rural residents were examined with chi-square cross tabulation for categorical variables and independent samples t-test for continuous variables. Multiple linear regression and logistic regression models were then applied to explore differences in health behaviors and self-reported chronic disease based on residence location. Model 1 was adjusted for age, sex, and province of residence. Model 2 was further adjusted for ethnicity, education, marital status, fruit and vegetable intake, smoking, alcohol use, multimorbidity, BMI, and total PA as applicable. ${ }^{18}$ The same modelling procedure was used to determine differences in measures of body adiposity. Participants living in urban areas were chosen as the reference group. When smoking, alcohol use, and PA were treated as the confounding factors in the multivariable regression analyses, they were kept as the categorical variables with multiple categories as shown in Table 1 . Whereas, when they were considered as our study outcomes, they were dichotomized as binary variables (yes/no). For example, current smokers vs. non-smokers and former smokers, regular and habitual drinkers vs. not regular and habitual drinkers, and those who engaged in high levels of PA vs those who engaged in low-to-moderate levels.

\section{Results}

Characteristics of both urban and rural groups are presented in Table 1 . Over $40 \%$ of the study sample had a university education and the majority were female (71.4\%), white (91\%), 
was married or had a partner $(81 \%)$. The average age and BMI of this sample was 54.0 years and $28.3 \mathrm{~kg} / \mathrm{m}^{2}$ respectively. Approximately $35 \%$ of participants that were coded with PCCF+ were classified as rural residents. Chi-square and t-test analyses revealed that rural residents were more likely than urban residents to be female $(P<.001)$, married $(P<.001)$, and older $(P<.001)$, but less likely to have completed higher education degrees $(P<.001)$.

\section{Rural-Urban Disparities in Health Behaviors}

Chi-square and t-test analyses revealed significant differences between rural and urban residents for frequency of alcohol consumption $(P<.001)$, PA level $(P<.001)$, and sitting time/sedentary behaviors $(P<.001)$. Differences in smoking behavior $(P=.352)$, multimorbidity $(P=.617)$, and fruit and vegetable intake $(P=.380)$ were non-significant.

Multiple logistic regression analyses showed that when compared to urban participants, rural residents were significantly less likely to be regular or habitual drinkers (OR 0.83 [95\% CI 0.78 to 0.89$])$. Rural residents were more likely to be highly active (1.19 [1.11 to 1.27$])$ but were more likely to be classified as obese (1.13 [1.05 to 1.21$])$ and present with abdominal obesity (1.08 [1.01 to 1.15]) (Model 1). Significant differences remained for drinking status (0.84 [0.79 to 0.90$])$, activity level (1.16 [1.08 to 1.24$])$, and obesity classification (1.12 [1.04 to 1.20]) after adjusting for potential confounding factors (Model 2). The likelihood for abdominal obesity became marginally significant (1.07 (1.00 to 1.14)). Likelihood of being a current smoker, having adequate fruit and vegetable intake, or having multimorbidity did not differ significantly between rural and urban residents in either Model 1 or Model 2. Detailed results can be found in Table 2 .

Rural-Urban Disparities in Body Adiposity 
Differences in various measures of body adiposity are presented in Table 3. Model 1 revealed that rural residents were significantly more likely to have higher BMI $(\beta(95 \% \mathrm{CI})=$ $0.50(0.32$ to 0.69$))$, body fat percentage $(0.40(0.12$ to 0.68$))$, and fat mass index $(0.34(0.20$ to 0.47)) than urban residents. After full adjustment in Model 2, significant differences remained in favor of the urban residents for BMI $(0.48(0.29$ to 0.66$))$, body fat percentage $(0.35$ (0.07 to $0.63))$ and fat mass index $(0.32(0.19$ to 0.46$))$. No differences in waist circumference were noted in either model.

\section{Discussion}

The aim of the current study was to describe and compare sociodemographic, PA and related health behaviors of Atlantic PATH participants based on rural or urban residency. Overall, we found that despite reporting greater levels of vigorous PA, rural residents were more likely to have higher BMI, body fat percentage, and fat mass index. These results partially support our hypothesis and much of the literature which suggests that rural residents generally have higher levels of obesity than urban residents. ${ }^{2,16-19}$ For example, studies using data from the US National Health and Nutrition Examination Survey (NHANES) found that obesity levels were higher among rural participants while PA levels were lower. ${ }^{16-19}$ Canadian data has similarly demonstrated higher rates of obesity in rural populations. ${ }^{2}$ In contrast, to these studies however, ${ }^{2,16-19}$ we did not find that rural participants were more likely to demonstrate an overall poorer health profile. Specifically, the finding of greater levels of vigorous PA, less sitting time, lower rates of regular or habitual alcohol consumption, and no significant differences in smoking behaviors, fruit and vegetable consumption, and multimorbidity was in contrast to the bulk of the existing literature. 
Although it is unclear why the results of Atlantic PATH are contrary to these studies, as noted by Fan et al., ${ }^{17}$ the variance in disparities between PA behaviors in rural and urban populations changes with the chosen method of PA measurement. Although a commonly utilized and well validated self-report measure of PA, relative to objective measures of PA (eg, accelerometers), the IPAQ has been reported to substantially overestimate PA levels. For example, a recent Statistics Canada study reported that $90 \%$ of respondents using the IPAQ-LF self-reported meeting the minimal PA guidelines (ie, 150 minutes of moderate-to-vigorous PA per week), while fewer than $30 \%$ met guidelines when assessed with an objective measure. ${ }^{43}$ Similarly, the short form has been demonstrated to overestimate PA by as much as $36-173 \%{ }^{44}$ However, as all Atlantic PATH participants completed either the long or short version of the IPAQ, overestimation of PA alone should not account for the difference in total PA between rural and urban residents seen in the current study. Notwithstanding, although the IPAQ has demonstrated acceptable measurement properties across diverse study samples, some studies have shown lower scale reliability in rural populations. ${ }^{35}$ Thus, while differences in PA may be the result of variability in the type and/or patterns of PA, other variables (ie, education, culture) may also play a role in how participants interpret and respond to items in the questionnaire. ${ }^{35,45}$ Interestingly, the study by Fan and colleagues ${ }^{17}$ reported that accelerometry data showed that rural participants were less active than urban residents; however, when subjective measures were used, rural residents reported more total PA than urban residents. In contrast to the current study which suggests that rural residents engage in more vigorous PA, Fan et al. ${ }^{17}$ found that rural residents appeared to engage in less high-intensity PA, with the primary difference in total PA being attributed to the higher reporting of domestic activities. Similarly, Patterson et al. ${ }^{18}$ noted that men living in non-urban areas reported an average of $19 \%$ more minutes of total PA 
per week ( $8 \%$ after adjusting for individual and socioeconomic factors). However, they also found that rural residents reported lower active transportation and leisure-time activities, but higher levels of domestic and occupational activities than their urban counterparts; suggesting that rural residents may acquire PA in different activity domains than urban residents. ${ }^{18}$ Although both Befort et al. ${ }^{16}$ and Trivedi et al. ${ }^{19}$ reported that rural residents were less likely to meet PA guidelines than those dwelling in urban areas, the measure of PA in both studies was limited to recreational PA. Notably, a recent study by Robertson and colleagues ${ }^{46}$ found that rural residents engage in higher levels of total PA, but less recreational PA. Unique to this study was the inclusion of muscular strengthening activities. As resistance training has been demonstrated to play an important role in weight management, the finding that rural residents were less likely to participate in leisure-time resistance training is an important area of future study. Moreover, while research has shown occupational and domestic PA can reduce the risk of some chronic diseases, recreational or leisure-time PA has been suggested to confer greater and potentially unique health benefits. ${ }^{47}$ As we combined data from both the long- and short-form IPAQ measures, thus necessitating the aggregation of domain-specific measures and the use of categorical indicators of total PA (ie, total vigorous, moderate, low PA), we were not able to distinguish where, if any domain specific differences might be within the current study. While this represents an area of need of further study, our findings support previous work which suggests that that rural residents may in engage in more total overall $\mathrm{PA}^{17,46}$; however, the method (ie, objective vs. subjective, domain specific self-report) of assessment of PA is likely to substantially influence study findings.

Although the PA literature is somewhat mixed and perhaps ultimately dependent on the measure used to capture PA, the finding of higher rates of obesity in rural participants is 
consistent with the bulk of the literature. However, the finding of less sedentary behavior (ie, sedentary sitting time), lower rates of regular and habitual alcohol use, and the lack of pronounced differences in smoking, fruit and vegetable intake, and multimorbidity was unexpected. When individual health behaviors and socio-economic and demographic factors were included in the multivariate analyses of obesity, rural residents continued to be at greater risk of obesity. These findings suggest, as with PA, that the outcome is likely influenced by the measure itself, self-report biases, and/or additional factors beyond those examined in this study

\section{Strengths and Limitations}

Atlantic PATH is the largest cohort study in the region and with the other CPTP cohorts, is the largest health cohort in Canada. However, limitations of this study include the crosssectional nature of the data, using self-report measures of health behaviors and chronic disease incidence, and the potential selection bias of recruitment methods. Atlantic PATH had recently completed recruitment, and as such, only baseline data was available for analyses; however, future follow-up measures will permit further observations. Additionally, provincial level health data will be linked Atlantic PATH which will allow for objective determination of health care utilization and chronic disease status. During recruitment, this study was clearly a health-related study and therefore, it is possible that the sample recruited were more motivated and in general, healthier than the average population. The study population was also wealthier and more educated than the average population. ${ }^{32}$ Moreover, while Atlantic PATH made concerted efforts to recruit rural residents (eg, mobile clinics, mailed study packs), our cohort is not fully representative of the rural population of the Atlantic provinces.

\section{Conclusions}


Though some of our results differ from previous literature, the majority of research to date has not been conducted within the Canadian context. While not fully representative of the Atlantic Canadian population, this study adds important insight to a growing body of literature which has explored the rural-urban disparities in health behaviors and outcomes in a geographically and culturally diverse Canadian setting.

Consistent with previous literature in developed countries, the current study found that rural residents were more likely to be obese. ${ }^{1,4,10,13,14,16,18,19,22}$ Interestingly, we found this relationship despite total PA levels, sedentary behaviors, and regular and habitual alcohol consumption favoring the rural participants. Follow-up assessments from Atlantic PATH participants will provide important data to help confirm or refute these findings and better elucidate the prospective association between health behaviors, anthropometric measures (eg, BMI, abdominal obesity), and rurality. The consideration and addition of objective measures of health indices (ie, PA) and neighborhood characteristics (eg, green space, retail and services, and walkability scores) would add a level of detail that would be valuable in future research. 


\section{References}

1. Wanner M, Richard A, Martin B, Faeh D, Rohrmann S. Associations between self-reported and objectively measured physical activity, sedentary behavior and overweight/obesity in NHANES 2003-2006. Int J Obes (Lond). 2017;41:186-193.

2. Mitura V, Bollman RD. The health of rural Canadians: A rural-urban comparison of health indicators. Rural and Small Town Canada Analysis Bulletin [serial online]. 2003;4:November $27,2018$.

3. Duncan MJ, Vandelanotte C, Caperchione C, Hanley C, Mummery WK. Temporal trends in and relationships between screen time, physical activity, overweight and obesity. BMC Public Health. 2012;12:1060-2458-12-1060.

4. Flint E, Cummins S. Active commuting and obesity in mid-life: cross-sectional, observational evidence from UK Biobank. Lancet Diabetes Endocrinol. 2016;4:420-435.

5. Shibata AI, Oka K, Sugiyama T, Salmon JO, Dunstan DW, Owen N. Physical Activity, Television Viewing Time, and 12-Year Changes in Waist Circumference. Med Sci Sports Exerc. 2016;48:633-640.

6. Bischoff SC, Boirie Y, Cederholm T, et al. Towards a multidisciplinary approach to understand and manage obesity and related diseases. Clin Nutr. 2017;36:917-938.

7. Zheng Y, Manson JE, Yuan C, et al. Associations of Weight Gain From Early to Middle Adulthood With Major Health Outcomes Later in Life. JAMA. 2017;318:255-269. 8. Flint E, Cummins S, Sacker A. Associations between active commuting, body fat, and body mass index: population based, cross sectional study in the United Kingdom. BMJ. 2014;349:g4887. 
9. Kim RB, Phillips A, Herrick K, et al. Physical activity and sedentary behavior of cancer survivors and non-cancer individuals: results from a national survey. PLoS One. 2013;8:e57598. 10. Yu E, Rimm E, Qi L, et al. Diet, Lifestyle, Biomarkers, Genetic Factors, and Risk of Cardiovascular Disease in the Nurses' Health Studies. Am J Public Health. 2016;106:1616-1623. 11. Ferguson C, Birch KM, Garnham JO, Witte KK. Exercise training: The under developed elixir vitae of chronic disease? Diab Vasc Dis Res. 2017;14:113-115.

12. Park S. Pathways linking obesity to health-related quality of life. Qual Life Res. 2017;26:2209-2218.

13. Kim DD, Basu A. Estimating the Medical Care Costs of Obesity in the United States: Systematic Review, Meta-Analysis, and Empirical Analysis. Value Health. 2016;19:602-613. 14. Wang YC, McPherson K, Marsh T, Gortmaker SL, Brown M. Health and economic burden of the projected obesity trends in the USA and the UK. Lancet. 2011;378:815-825.

15. Egger G, Dixon J. Beyond obesity and lifestyle: a review of 21 st century chronic disease determinants. Biomed Res Int. 2014;2014:731685.

16. Befort CA, Nazir N, Perri MG. Prevalence of obesity among adults from rural and urban areas of the United States: findings from NHANES (2005-2008). J Rural Health. 2012;28:392397.

17. Fan JX, Wen M, Kowaleski-Jones L. Rural-urban differences in objective and subjective measures of physical activity: findings from the National Health and Nutrition Examination Survey (NHANES) 2003-2006. Prev Chronic Dis. 2014;11:E141.

18. Patterson KA, Cleland V, Venn A, Blizzard L, Gall S. A cross-sectional study of geographic differences in health risk factors among young Australian adults: the role of socioeconomic position. BMC Public Health. 2014;14:1278-2458-14-1278. 
19. Trivedi T, Liu J, Probst J, Merchant A, Jhones S, Martin AB. Obesity and obesity-related behaviors among rural and urban adults in the USA. Rural Remote Health. 2015;15:3267.

20. Kondro W. Health of rural Canadians lags behind urban counterparts. CMAJ. 2006;175:1195.

21. Blanchard C, Rainham D, McSweeney J, et al. Community SES, perceived environment, and physical activity during home-based cardiac rehabilitation: is there a need to consider the urban vs. rural distinction? J Urban Health. 2012;89:285-295.

22. Sugiyama T, Niyonsenga T, Howard NJ, et al. Residential proximity to urban centres, localarea walkability and change in waist circumference among Australian adults. Prev Med. 2016;93:39-45.

23. Bhatnagar A. Environmental Determinants of Cardiovascular Disease. Circ Res. 2017;121:162-180.

24. Perrin AJ, Caren N, Skinner AC, Odulana A, Perrin EM. The unbuilt environment: culture moderates the built environment for physical activity. BMC Public Health. 2016;16:1227-0163866-3.

25. Booth KM, Pinkston MM, Poston WS. Obesity and the built environment. J Am Diet Assoc. 2005;105:S110-7.

26. Saint-Jacques N, Dewar R, Cui Y, Parker L, Dummer TJ. Premature mortality due to social and material deprivation in Nova Scotia, Canada. Int J Equity Health. 2014;13:94-014-0094-2.

27. Prince SA, Reed JL, McFetridge C, Tremblay MS, Reid RD. Correlates of sedentary behaviour in adults: a systematic review. Obes Rev. 2017;18:915-935.

28. Statistics Canada. Table 17-10-0118-01 Selected population characteristics, Canada, provinces and territories. Available at:

https://www150.statcan.gc.ca/t1/tbl1/en/cv.action?pid=1710011801. Accessed 07/23, 2018. 
29. Hayward K, Colman R. Addressing inequity and chronic disease in Atlantic Canada. A discussion paper. Health Canada; 2003. Available from:

http://www.gpiatlantic.org/pdf/health/inequity.pdf.

30. Hayward K, Colman R. The Tides of Change: Addressing inequity and chronic disease in Atlantic Canada. A discussion paper. Health Canada; 2003. Available from:

http://www.gpiatlantic.org/pdf/health/inequity.pdf.

31. Dummer TJB, Awadalla P, Boileau C, et al. The Canadian Partnership for Tomorrow Project: a pan-Canadian platform for research on chronic disease prevention. CMAJ. 2018;190:E710-E717.

32. Sweeney E, Cui Y, DeClercq V, et al. Cohort Profile: The Atlantic Partnership for Tomorrow's Health (Atlantic PATH) Study. International Journal of Epidemiology. 2017. 33. Keats MR, Cui Y, DeClercq V, et al. Multimorbidity in Atlantic Canada and association with low levels of physical activity. Prev Med. 2017;105:326-331.

34. Grundy SM, Cleeman JI, Daniels SR, et al. Diagnosis and management of the metabolic syndrome: an American Heart Association/National Heart, Lung, and Blood Institute scientific statement: Executive Summary. Crit Pathw Cardiol. 2005;4:198-203.

35. Craig CL, Marshall AL, Sjostrom M, et al. International physical activity questionnaire: 12country reliability and validity. Med Sci Sports Exerc. 2003;35:1381-1395.

36. Hagstromer M, Oja P, Sjostrom M. The International Physical Activity Questionnaire (IPAQ): a study of concurrent and construct validity. Public Health Nutr. 2006;9:755-762. 
37. International Physical Activity Questionnaire. Available at: https://sites.google.com/site/theipaq/home.

38. Yu ZM, De Clercq V, Cui Y, et al. Fruit and vegetable intake and obesity among populations in Eastern Canada: The Atlantic Partnership for Tomorrow's Health study. BMJ Open. 2018. doi:10.1136/bmjopen-2017-018060.

39. Statistics Canada. Canadian Community Health Survey, Cycle 1.1. Ottawa: Statistics Canada; 2001. Available from:

http://www23.statcan.gc.ca/imdb/p2SV.p1?Function=getInstanceList\&Id=329241.

40. Statistics Canada. Canadian Community Health Survey, Cycle 3.1. Ottawa: Statistics Canada; 2005. Available from:

http://www23.statcan.gc.ca/imdb/p2SV.pl?Function=getInstanceList\&Id=329241. 41. Statistics Canada. Canadian Health Measures Survey, Cycle 1. Ottawa: Statistics Canada; 2007-2009. Available from:

http://www23.statcan.gc.ca/imdb/p3Instr.pl?Function=getInstrumentList\&Item_Id=64294\&UL= $1 \mathrm{~V}$.

42. Statistics Canada. Canada Tobacco Use Monitoring Survey. Ottawa: Statistics Canada; 2007. Available from: http://www23.statcan.gc.ca/imdb/p2SV.pl?Function=getSurvey\&Id=44877. 43. Garriguet D, Tremblay S, Colley RC. Comparison of Physical Activity Adult Questionnaire results with accelerometer data. Health Rep. 2015;26:11-17.

44. Lee PH, Macfarlane DJ, Lam TH, Stewart SM. Validity of the International Physical Activity Questionnaire Short Form (IPAQ-SF): a systematic review. Int J Behav Nutr Phys Act. 2011;8:115-5868-8-115. 
45. Lachat CK, Verstraeten R, Khanh le NB, et al. Validity of two physical activity questionnaires (IPAQ and PAQA) for Vietnamese adolescents in rural and urban areas. Int $J$ Behav Nutr Phys Act. 2008;5:37-5868-5-37.

46. Robertson MC, Song J, Taylor WC, Durand CP, Basen-Engquist KM. Urban-Rural Differences in Aerobic Physical Activity, Muscle Strengthening Exercise, and Screen-Time Sedentary Behavior. J Rural Health. 2018.

47. Holtermann A, Krause N, van der Beek AJ, Straker L. The physical activity paradox: six reasons why occupational physical activity (OPA) does not confer the cardiovascular health benefits that leisure time physical activity does. Br J Sports Med. 2018;52:149-150. 
Table 1 Characteristics of Study Participants ${ }^{\mathrm{a}}$

\begin{tabular}{|c|c|c|c|c|c|}
\hline \multirow[b]{2}{*}{ Age, years M(SD) ${ }^{\dagger}$} & \multicolumn{2}{|c|}{$\begin{array}{c}\text { Urban } \\
(n=11,132)\end{array}$} & \multicolumn{2}{|c|}{$\begin{array}{c}\text { Rural } \\
(\mathrm{n}=5922)\end{array}$} & \multirow{2}{*}{$\begin{array}{c}\text { p-values } \\
<.001\end{array}$} \\
\hline & 53.7 & $(9.0)$ & 54.2 & $(8.7)$ & \\
\hline Female, $\mathbf{n}(\%)^{\phi}$ & 7761 & $(69.7)$ & 4330 & $(73.1)$ & $<.001$ \\
\hline Province, $\mathbf{n}(\%)^{\phi}$ & & & & & $<.001$ \\
\hline Nova Scotia & 6359 & $(57.1)$ & 3514 & $(59.3)$ & \\
\hline New Brunswick & 2999 & $(26.9)$ & 1738 & $(29.3)$ & \\
\hline Newfoundland and Labrador & 1468 & $(13.2)$ & 442 & $(7.5)$ & \\
\hline Prince Edward Island & 306 & $(2.7)$ & 228 & $(3.9)$ & \\
\hline Ethnicity, $\mathbf{n}(\%)^{\phi}$ & & & & & .003 \\
\hline White & 10131 & $(91.0)$ & 5346 & $(90.3)$ & \\
\hline Non-white & 637 & $(5.7)$ & 323 & $(5.5)$ & \\
\hline DNK/PNA & 364 & $(3.3)$ & 253 & $(4.3)$ & \\
\hline Education, $\mathbf{n}(\%)^{\Phi}$ & & & & & $<.001$ \\
\hline Less than high school & 1684 & $(15.1)$ & 1245 & $(21.0)$ & \\
\hline College level & 4194 & $(37.7)$ & 2599 & $(43.9)$ & \\
\hline University level or higher & 5223 & $(46.9)$ & 2059 & $(34.8)$ & \\
\hline DNK/PNA & 31 & $(0.3)$ & 19 & $(0.3)$ & \\
\hline Marital status, $\mathbf{n}(\%)^{\Phi}$ & & & & & $<.001$ \\
\hline Married or living together & 8542 & $(76.7)$ & 5047 & $(85.2)$ & \\
\hline $\begin{array}{l}\text { Single, divorced, separated, or } \\
\text { widowed }\end{array}$ & 2562 & $(23.0)$ & 865 & $(14.6)$ & \\
\hline DNK/PNA & 28 & $(0.3)$ & 10 & $(0.2)$ & \\
\hline Smoking status, $\mathbf{n}(\%)^{\phi}$ & & & & & .352 \\
\hline Never & 5722 & $(51.4)$ & 2976 & $(50.3)$ & \\
\hline Former & 4451 & $(40.0)$ & 2420 & $(40.9)$ & \\
\hline Current & 892 & $(8.0)$ & 481 & $(8.1)$ & \\
\hline DNK/PNA & 67 & $(0.6)$ & 45 & $(0.8)$ & \\
\hline Alcohol use, $n(\%)^{\Phi}$ & & & & & $<.001$ \\
\hline Abstainer & 1013 & $(9.1)$ & 671 & $(11.3)$ & \\
\hline Occasional drinker & 4420 & $(39.7)$ & 2494 & $(42.1)$ & \\
\hline Regular drinker & 3551 & $(31.9)$ & 1684 & $(28.4)$ & \\
\hline Habitual drinker & 1965 & $(17.7)$ & 959 & $(16.2)$ & \\
\hline DNK/PNA & 183 & $(1.6)$ & 114 & $(1.9)$ & \\
\hline Multimorbidity $^{\mathrm{b}}, \mathbf{n}(\%)^{\Phi}$ & & & & & .617 \\
\hline None & 3983 & $(35.8)$ & 2082 & $(35.2)$ & \\
\hline One & 3652 & $(32.8)$ & 1983 & $(33.5)$ & \\
\hline Two or more & 3497 & $(31.4)$ & 1857 & $(31.4)$ & \\
\hline Physical activity level ${ }^{\mathbf{c}}, \mathbf{n}(\%)^{\Phi}$ & & & & & $<.001$ \\
\hline Low activity & 3770 & $(33.9)$ & 1913 & $(32.3)$ & \\
\hline Moderate activity & 3939 & $(35.4)$ & 1944 & $(32.8)$ & \\
\hline High activity & 3423 & $(30.7)$ & 2065 & $(34.9)$ & \\
\hline Body weight, kg, M(SD) ${ }^{\dagger}$ & 78.0 & $(17.5)$ & 78.9 & (19.1) & .004 \\
\hline Body height, cm, M(SD) ${ }^{\dagger}$ & 166.5 & $(7.2)$ & 166.2 & $(7.0)$ & .037 \\
\hline
\end{tabular}




\begin{tabular}{|c|c|c|c|c|c|}
\hline Body mass index, $\mathrm{kg} / \mathrm{m}^{2}, \mathrm{M}(\mathrm{SD})^{\dagger}$ & 28.1 & $(5.9)$ & 28.5 & $(6.4)$ & $<.001$ \\
\hline Waist Circumference, $\mathbf{c m}, \mathbf{M}(\mathbf{S D})^{\dagger}$ & 93.2 & $(14.7)$ & 93.6 & $(14.8)$ & .137 \\
\hline Percentage fat mass, $\%, M(S D)^{\dagger}$ & 33.4 & $(9.1)$ & 33.9 & $(9.3)$ & .004 \\
\hline Fat mass index, $\mathrm{kg} / \mathrm{m}^{2}, \mathbf{M}(\mathrm{SD})^{\dagger}$ & 9.7 & (4.3) & 10.0 & (4.7) & $<.001$ \\
\hline Fat free mass index, $\mathbf{k g} / \mathrm{m}^{2}, \mathbf{M}(\mathrm{SD})^{\dagger}$ & 18.4 & (3.1) & 18.5 & (3.3) & .126 \\
\hline $\begin{array}{l}\text { Fruit and vegetable intake, } \\
\text { serving/day, } M(\mathrm{SD})^{\dagger}\end{array}$ & 5.4 & $(2.7)$ & 5.5 & $(2.6)$ & .380 \\
\hline Sitting time, hours/day, $M(\mathrm{SD})^{\dagger}$ & 5.8 & $(2.9)$ & 5.4 & $(2.7)$ & $<.00$ \\
\hline
\end{tabular}

DNK $=$ Do Not Know; PNA $=$ Prefer Not to Answer.

$f=\mathrm{t}$-test; $\phi=$ chi square.

${ }^{a}$ Data are means (standard deviation) and number of participants (percentage).

bSelf-reported chronic conditions.

'Data driven tertiles 
Table 2 Differences in the Prevalence of Health Behaviors, Obesity, and Multimorbidity Between Participants Living in Urban and Rural Areas

\begin{tabular}{|c|c|c|c|c|c|}
\hline \multicolumn{6}{|c|}{ ORs (95\% CIs) } \\
\hline & Case $/ \mathbf{n}$ & Urban & Case/n & & Rural \\
\hline \multicolumn{6}{|c|}{ Current smoker } \\
\hline Model 1 & $892 / 11132$ & Reference & $481 / 5922$ & 1.03 & $(0.92,1.16)$ \\
\hline Model 2 & & Reference & & 1.00 & $(0.89,1.13)$ \\
\hline \multicolumn{6}{|c|}{ Regular or habitual alcohol drinker } \\
\hline Model 1 & $5516 / 11132$ & Reference & $2643 / 5922$ & 0.83 & $(0.78,0.89)$ \\
\hline Model 2 & & Reference & & 0.84 & $(0.79,0.90)$ \\
\hline \multicolumn{6}{|c|}{ High physical activity } \\
\hline Model 1 & $3423 / 11132$ & Reference & $2065 / 5922$ & 1.19 & $(1.11,1.27)$ \\
\hline Model 2 & & Reference & & 1.16 & $(1.08,1.24)$ \\
\hline \multicolumn{6}{|c|}{ Adequate fruit and vegetable intake } \\
\hline Model 1 & $3324 / 11132$ & Reference & $1763 / 5922$ & 0.98 & $(0.91,1.05)$ \\
\hline Model 2 & & Reference & & 1.01 & $(0.94,1.08)$ \\
\hline \multicolumn{6}{|l|}{ Obesity } \\
\hline Model 1 & $3523 / 11132$ & Reference & $1975 / 5922$ & 1.13 & $(1.05,1.21)$ \\
\hline Model 2 & & Reference & & 1.12 & $(1.04,1.20)$ \\
\hline \multicolumn{6}{|c|}{ Abdominal obesity } \\
\hline Model 1 & $5571 / 11132$ & Reference & $3125 / 5922$ & 1.08 & $(1.01,1.15)$ \\
\hline Model 2 & & Reference & & 1.07 & $(1.00,1.14)$ \\
\hline \multicolumn{6}{|c|}{ Multimorbidity } \\
\hline Model 1 & $3497 / 11132$ & Reference & $1857 / 5922$ & 0.97 & $(0.90,1.04)$ \\
\hline Model 2 & & Reference & & 0.94 & $(0.88,1.01)$ \\
\hline
\end{tabular}

Categories presented in Table 1 were used in the final regression models.

Model 1, adjusted for age, sex, and province.

Model 2, further adjusted for ethnicity, education, marital status, adequate fruit \& vegetable intake, smoking, alcohol use, multimorbidity, BMI, abdominal obesity, and total physical activity (where applicable) based on model 1. 
Table 3. Differences in Body Adiposity Measures Between Participants Living in Urban and Rural Areas

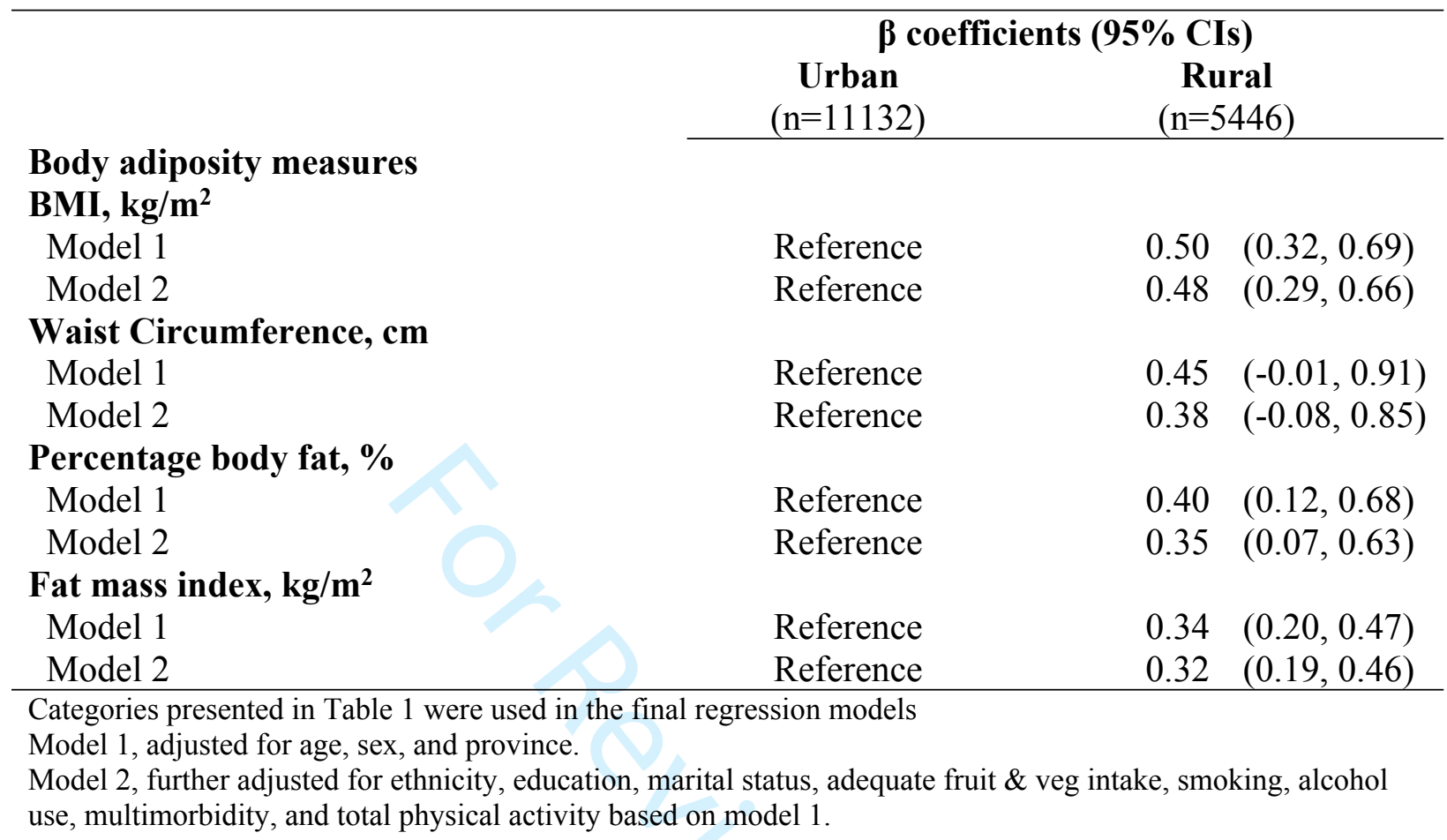

